\title{
Categorization influences illusory conjunctions
}

\author{
MICHAEL ESTERMAN \\ University of California, Berkeley, Califormia \\ and Veterans Administration Medical Research, Martinez, California \\ WILLIAM PRINZMETAL \\ University of California, Berkeley, California \\ and \\ LYNN ROBERTSON \\ University of Califormia, Berkeley, California \\ and Veterans Administration Medical Research, Martinez, California
}

\begin{abstract}
Illusory conjunctions (ICs) provide evidence for a binding problem that must be resolved in vision. Objects that are perceptually grouped are more likely to have their features erroneously conjoined. We examined whether semantic grouping, determined by category membership (letter vs. number), also influences illusory conjunction rates. Participants were instructed to detect an "L" or a "7" among briefly presented character strings and to report its color. Despite high shape discrimination accuracy, participants often made color conjunction errors, reporting instead the color of a distractor character, "O". This distractor could be ambiguously interpreted as a letter or a number. The status of the "O" was determined by other noncolored flanker characters, which were either letters or numbers. When both the target and flankers were of the same category, participants made more ICs than when the target and flankers were of different categories. This finding demonstrates that alphanumeric categorization can precede and subsequently influence binding.
\end{abstract}

Illusory conjunctions (ICs) occur when viewers incorrectly perceive a feature of one item, such as color, as belonging to another item in a display. Originally reported by Treisman and Schmidt (1982), ICs provide evidence for a binding problem in vision. Since then, further evidence has accumulated demonstrating that ICs can occur in a variety of situations and that many visual and cognitive factors can constrain when these binding errors are likely to occur.

One of the most powerful constraints on binding is attention. When spatial attention is distracted by a secondary task, ICs occur more frequently (Treisman \& Schmidt, 1982). This finding made a critical contribution to the development of feature integration theory (FIT), which proposed that individual features are registered in parallel and that conjoining features requires focused attention. Subsequent research found that binding errors can occur in the periphery, even without a distracting task, suggesting that poor spatial resolution or localizability hinders the visual system's ability to bind features accurately (Prinzmetal, Henderson, \& Ivry, 1995). Neuropsychological evidence

Correspondence concerning this article should be addressed to M. Esterman, Department of Psychology, University of California, 3210 Tolman Hall, Room 1650, Berkeley, CA 94720-1650 (e-mail: esterman@, uclink.berkeley.edu). corroborates this perspective. Reports of increased ICs following brain injury have been concurrent with a loss of spatial abilities (Arguin, Cavanagh, \& Joanette, 1994; Bernstein \& Robertson, 1998; Cohen \& Rafal, 1991; FriedmanHill, Robertson, \& Treisman, 1995; Robertson, Treisman, Friedman-Hill, \& Grabowecky, 1997; Ward, Danziger, Owen, \& Rafal, 2002).

Early visual processing not only appears to register features in parallel, but also organizes these features into perceptual groups and proto-objects represented as bundles of features (Wolfe \& Bennett, 1997). These grouping processes have also been shown to constrain feature binding: ICs are more likely to occur within items grouped by proximity, similarity, or continuation (Cohen \& Ivry, 1989; Ivry \& Prinzmetal, 1991; Prinzmetal, 1981). Treisman and colleagues also found that figure-ground organization influences binding in such a way that more ICs are made within the figure than between figure and ground (Treisman, 1988).

Linguistic processes also influence the likelihood of making a binding error. For instance, more ICs occur within letter strings that form real words, or pronounceable nonwords, than within random letter strings (Prinzmetal \& Millis-Wright, 1984). Furthermore, grouping based on word structure, such as orthography and morphology, can also influence binding. For example, when single words 
with letters printed in various colors were presented, ICs of letters and colors within syllables were more likely than ICs between letters and colors in different syllables (Prinzmetal, Hoffman, \& Vest, 1991). Prinzmetal and Keysar (1989) demonstrated that if context shifted syllable structure, it also affected ICs. In that study, Hebrew words were presented without vowels (as is typical in Hebrew) in such a way that out of context, words with the same spelling could have different pronunciations and syllabic divisions. When a contextual cue preceded and thus biased the interpretation of the word, more ICs occurred between the letters within the same syllable than between the letters in different syllables. This study demonstrates that linguistic structure constrains feature binding, independently of the physical characteristics of the stimuli.

Collectively, these studies indicate that processing of letter and word form can influence binding, perhaps reflecting the automatic nature of grouping letters into words. It nevertheless remains unclear whether semantic or conceptual categories, such as the meaning of a word or object, have such an influence on binding. At least one set of experiments has addressed this issue. Treisman (1988) presented displays created from colored shapes that were labeled either by noncommittal names (ellipse, triangle) or by constrained names whose meaning suggested a color (lake, carrot). Constrained names presented in their expected colors (e.g., blue lake) eliminated conjunction errors. However, when presented in nontypical colors (e.g., red lake), constraining labels did not lead ICs to "correct" the object to its expected color. Treisman concluded that although expectancy seemed to help participants rule out inappropriate conjunctions, this occurred after binding took place, suggesting that semantics does not influence the binding process itself.

The present study addressed whether objects with similar meaning can influence grouping and, subsequently, binding. If so, more ICs should occur between items that belong to the same category than between items that belong to different categories. In this study, the categories were letters and numbers. We took advantage of the fact that the character " $\mathrm{O}$ " can be interpreted as either a letter or a number, depending on the context (Jonides \& Gleitman, 1972). We manipulated the context to determine whether the color of the "O" would be more likely conjoined with another letter when "O" was categorically biased as a letter, and conversely more likely to be conjoined with another number when "O" was categorically biased as a number. We hypothesized that characters appearing to belong to the same alphanumeric category would be semantically grouped and that this would increase the probability of making an illusory conjunction. This result would unambiguously demonstrate an influence of semantics on feature binding.

\section{METHOD}

\section{Participants}

Fourteen participants were recruited: 12 were undergraduate psychology students, and 2 were staff members within the psychology department. All gave informed consent before participation. All the participants had normal or corrected-to-normal acuity and normal color vision. Their ages ranged from 19 to 28 , and all were naive to the experimental hypotheses. English was their first language.

\section{Apparatus}

Stimuli were presented with a Macintosh G4 Powerbook computer. The software package, Psychlab, was used to present stimuli with millisecond accuracy. Responses were made verbally after each trial and recorded by the experimenter. The participant's chair was positioned so that eye level was at midline and was maintained at a distance of approximately $46 \mathrm{~cm}$ from the computer screen.

\section{Stimuli}

Stimuli consisted of a horizontal string of four characters printed in 36-point Geneva font (see Figure 1). This string was briefly presented in one quadrant on a black screen. The two inner characters were colored, one being the target, and the other the distractor. The target was either an "L" or a "7" (a 180 rotated "L"). The distractor was always an ambiguous letter/number "O" (a capital letter "O"). The target and distractor were always in different colors. The colors used were highly distinguishable red (RGB\%: 87,4,3), green (RGB\%: $13,72,9)$, and blue (RGB\%: 1,1,83). The two outer or flanker characters were either both "S"s or both " 8 "s and were always white. Characters subtended approximately $.79^{\circ} \times 1.19^{\circ}$ of visual angle.

These characters yielded 48 possible character strings (two targets, two target positions, two flankers, and three colors). The different combinations created two main conditions: (1) In the same category condition, the flankers were consistent with the distractor "O" interpreted as being of the same alphanumeric category as the target (see Figure 1, left) and (2) in the different category condition, the flankers were consistent with the "O" interpreted as being of a different alphanumeric category than the target (see Figure 1, right).

Eccentricity of the inner edge of the character string began at $5.27^{\circ}$ from central fixation (in one of the four quadrants of the screen). The characters were evenly spaced $1 \mathrm{~cm}$ apart. Given the experimental procedure subsequently described, eccentricity was increased to $7.01^{\circ}$ or $8.74^{\circ}$ for certain individuals.

\section{Procedure}

The participants were instructed to fixate a central white dot. The dot changed to a cross $500 \mathrm{msec}$ before the character string appeared. The participants were instructed to maintain fixation on the cross. The character string flashed for $150 \mathrm{msec}$ randomly in one of the four locations, and the screen then turned black. The participants were asked to identify the target character ("L" or "7"), as well as to identify what color they thought the target character had been (red, green, or blue). Following their verbal report, another trial began approximately $1,000 \mathrm{msec}$ after the experimenter entered the response. Unlike in full-report paradigms examining ICs, the memory requirements of this task were negligible, given that there was
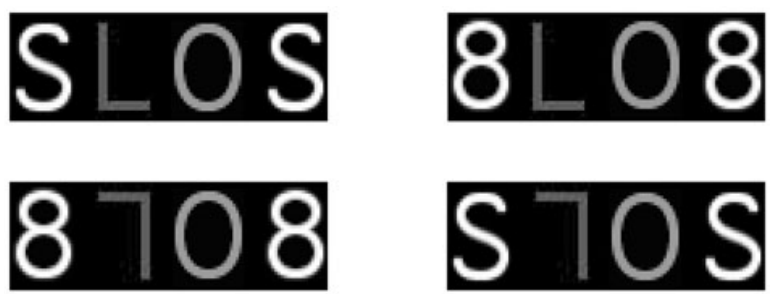

Figure 1. Left: Sample stimuli for same-category condition for letter target "L" (top) and number target "7" (bottom). Right: Sample stimuli for different-category condition for letter target "L" (top) and number target "7" (bottom). 
minimal memory load and no delay period between display and response.

The participants were given 4 practice trials, in which character strings were presented for $1,000,800,600$, and $400 \mathrm{msec}$. The experiment consisted of 192 trials (4 blocks of 48 trials), with each possible character string appearing in each location. Each block was counterbalanced to contain the same number of each trial type, colors, and targets. Trials were pseudorandomized within each block.

In many illusory conjunction experiments, timing and eccentricity must be optimal for significant ICs to occur. If conditions are too difficult, numerous letter and color errors occur, in which case conjunction errors are not likely to be "true" ICs, but the result of guessing. To address this problem, criteria were devised to ensure an optimal number of conjunction errors and minimal numbers of other errors. For a block to be included in the data set, it had to reach the criterion of at least $15 \%$ conjunction errors ( 8 of 48 ) and less than $15 \%$ intrusion errors (misidentifying the target or reporting an absent color).

The initial block was performed at the innermost eccentricity, and stimulus presentation time was $150 \mathrm{msec}$. This was the easiest setting and was appropriate for most participants (8 of 14) throughout the experiment. If performance was worse than criterion (too many intrusion errors), the subsequent block was performed under the same conditions, since practice typically improved performance. If performance was better than criterion (too few conjunction errors), presentation time was reduced and eccentricity was increased in an alternating fashion as follows: $100 \mathrm{msec}, 7.01^{\circ}, 83 \mathrm{msec}, 8.74^{\circ}$, $67 \mathrm{msec}$. After four experimental blocks, the blocks that did not reach criterion were repeated in random order, with a maximum of eight total blocks per participant.

Two participants required reduced presentation time, and two others required increased eccentricity in order to reach criterion. One participant was excluded because he made very few errors and did not reach criterion even when eccentricity was maximized and presentation time was minimized. A second subject asked to terminate the experiment before completion because of eye discomfort. Thus, the results reported reflect 12 participants.

\section{RESULTS}

\section{Raw Response Data}

In the following discussion, the designations for response types are abbreviated in such a way that the first letter indicates whether the target character was identified correctly (C) or incorrectly (I). The second letter represents the reported color: the target color (T), the distractor color (D), or the other color not present in the display $(\mathrm{O})$. In addition to a correct response (CT), there were five different kinds of possible errors. If the participant correctly reported the target character but incorrectly reported the distractor color, this was a conjunction error (CD). Such trials are candidates for ICs. If a participant reported the correct target character but a color that was absent from the display, this was a color error (CO). Similarly, the participant might have incorrectly reported the target character but correctly reported the target color (IT), the distractor color (ID), or an absent color (IO). Response proportions for each of these response types are shown in Figure 2 for both the same- and differentcategory conditions. The participants had fewer correct responses (CTs) in the same condition than in the different condition $[t(11)=2.92, p<.01]$.

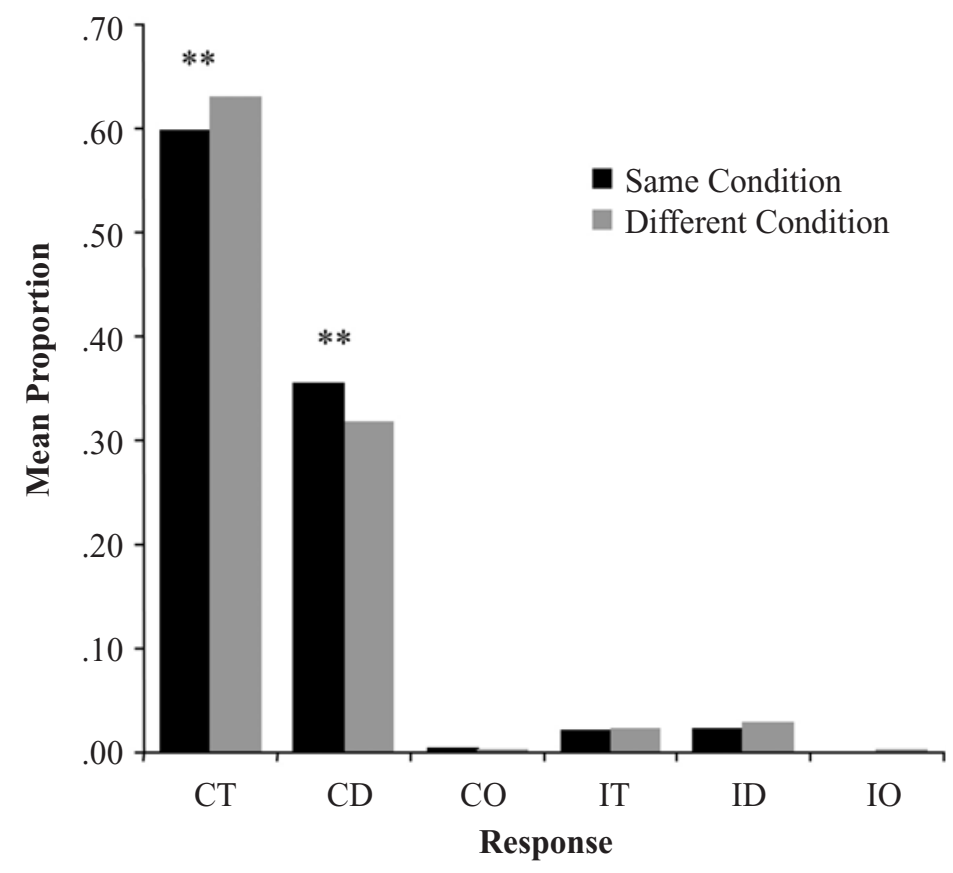

Figure 2. Mean proportions of all possible response types for same- and different-category conditions. CT, correct character, target color; $C D$, correct character, distractor color; $\mathrm{CO}$, correct character, other color; IT, incorrect character, target color; ID, incorrect character, distractor color; IO, incorrect character, other color. Asterisks denote significant differences between sameand different-category conditions $(p<.01)$. 
Of the error types, paired $t$ tests revealed that only proportion of conjunction errors (CDs) significantly differs across these two conditions $[t(11)=4.94, p<.001]$. All 12 participants had more conjunction errors in the same condition than in the different condition (mean difference $=.038, S E=.008$ ). Critically, this difference accounts for why participants made fewer correct responses in the same condition than in the different condition (mean difference $=.033, S E=.011$ ).

The raw data strongly suggest that the majority of these conjunction errors were due to errors in feature binding, and not errors in confusing the distractor ("O") with the target (Donk, 1999; Prinzmetal, Diedrichsen, \& Ivry, 2001). If CDs were due to confusing the distractor with the target, the participants would have reported the distractor color but would have had to guess the target character. Therefore, mistaking the distractor with the target would lead to an equal number of CDs as of IDs, which is far from the case (.34 and .05 , respectively). It can also be inferred from these data that conjunction errors were not due to misperceiving the target and/or distractor color, and therefore guessing the color. If this were the case, the number of color conjunctions would equal the number of color errors, but they do not (.34 and .003 , respectively).

\section{Conjunction Errors}

Because the large majority of conjunction errors (CDs in Figure 2) likely reflect correct perception of target features and errors in feature binding, conjunction error rates were subjected to a univariate analysis of variance, in order to further explore the differences between the same- and different-category conditions. Independent variables included target category (letter, number) and flanker category (letter, number). There was a significant interaction between target category and flanker category. As noted previously, when the target and flankers were of the same category, a higher rate of conjunction errors was observed between the target and the distractor " $O$ " than when the target and flankers were of different categories $[F(1,11)=$ $24.4, p<.001]$. Furthermore, this effect was present for both letter and number targets. When the target was an "L," participants made more conjunction errors when the flankers were "S"s $(M=.365, S E=.029)$ rather than " 8 "s $(M=.337, S E=.027)[F(1,11)=6.46, p<.05]$. In contrast, when the target was a "7," participants made more conjunction errors when the flankers were " 8 "s $(M=$ $.345, S E=.028)$ rather than "S"s $(M=.297, S E=.031)$ $[F(1,11)=19.70, p=.001]$. Thus, when the "O" was more likely to be categorized as a letter, its color was more likely to be conjoined with " $L$ " than with " 7 ," and when the "O" was more likely to be categorized as a number, its color was more likely to be conjoined with "7" than with "L."

\section{Model Analysis}

Different procedures have been developed to formally correct for guessing in illusory conjunction experiments. One of the most fruitful of these approaches has been multinomial modeling, which allows independent estima- tion of the probabilities of correctly perceiving features from the probability of correctly conjoining the same features (Ashby, Prinzmetal, Ivry, \& Maddox, 1996; Prinzmetal, Ivry, Beck, \& Shimizu, 2002). ${ }^{1}$ Adapting the model to the data from this experiment yielded four parameters. The first was the probability of perceiving the target character (TL). The second and third were the probabilities of perceiving the target color (TC) and distractor color (DC). The final parameter was the probability of properly conjoining the target color with the target character $(\alpha)$. In this model, $1-\alpha$ is the probability that the participant erroneously conjoined the distractor color with the target character. The model also required enumerating all ways in which each of the six possible response types could be reported. Thus, a probability tree was constructed, reflecting each parameter and the subsequent response. For example, a true illusory conjunction would occur if the participant correctly perceived the target character (TL), the target color (TC), and the distractor color (DC), and if the participant erroneously conjoined features $(1-\alpha)$. Although this would lead to a conjunction error (CD), other "false" ICs could also lead to a conjunction error, such as failing to perceive the target character $(1-\mathrm{TL})$, the target color $(1-\mathrm{TC})$, or the distractor color $(1-\mathrm{DC})$. On one sixth of these trials, the participant would have happened to guess the target character and distractor color and produced a conjunction error, but not a "true" illusory conjunction. We estimated these parameters with values that best fit the data for each individual participant. The bestfit measure used was $G^{2}$, but these results were replicated using a sum of squares error. Minimizing the $G^{2}$ led to the best estimation of the four parameters.

Table 1 summarizes the parameters estimated by the model and contrasts the actual data with the predictions of the model. These overall estimates are the result of averaging the model's parameters and predictions for each individual participant. Alpha rates were quite low, indicating that many of the CDs were true ICs. Paired $t$ tests revealed that although TL, TC, and DC did not differ between the same- and different-category conditions ( $p \mathrm{~s}>$ $.15), \alpha$ differed significantly $[t(11)=3.56, p<.01]$. This illustrates that when target and distractor were categorized as the same, their colors were more likely to be erroneously conjoined than when the target and distractor were categorized as different.

One criticism of the model is that it assumes that when participants do not perceive the target character, they nonetheless still know which of the two characters was the target. Alternatively, it may be that when participants do not perceive the target character, they do not even know which character was the target. When the model is adjusted to fit this alternative, estimates of TL, TC, and DC do not change significantly, while $\alpha$ does increase slightly, overall from .64 to $.66[t(23)=4.18, p<.001]$. Critically, however, the effect of category (increased $\alpha$ in the different condition vs. the same condition) does not change significantly; in fact, it increases slightly (.032 vs $.039, p>.1)$. It is likely that the true value is somewhere between these two 
Table 1

Actual and Predicted Response Proportions, as Well as Model Parameters With Estimated Values

\begin{tabular}{lcccc}
\hline & \multicolumn{2}{c}{ Response Proportion } & & \\
\cline { 2 - 3 } Response Type & Actual Value & Predicted Value & Model Parameter & Estimated Value \\
\hline & \multicolumn{5}{c}{ Same Condition } & & \\
CT & $.598^{* *}$ & $.592^{*}$ & $p(\mathrm{TL})$ & .913 \\
CD & $.355^{* *}$ & $.361^{* *}$ & $p(\mathrm{TC})$ & .989 \\
CO & .003 & .003 & $p(\mathrm{DC})$ & 1.00 \\
IT & .021 & .027 & $\alpha$ & $.622^{* *}$ \\
ID & .023 & .016 & & \\
IO & .000 & .000 & & \\
& & Different Condition & & \\
CT & $.631^{* *}$ & $.620^{*}$ & $p(\mathrm{TL})$ & .899 \\
CD & $.317^{* *}$ & $.327^{* *}$ & $p(\mathrm{TC})$ & .092 \\
CO & .002 & .002 & $p(\mathrm{DC})$ & $.655^{* *}$ \\
IT & .022 & .033 & $\alpha$ & \\
ID & .028 & .017 & & \\
IO & .001 & .000 & & \\
\hline
\end{tabular}

Note- CT, correct character, target color; $\mathrm{CD}$, correct character, distractor color; $\mathrm{CO}$, correct character, other color; IT, incorrect character, target color; ID, incorrect character, distractor color; IO, incorrect character, other color. Parameters included probability of perceiving the target character (TL), target color (TC), and distractor color (DC), as well as the probability of properly conjoining the target color with the target character $(\alpha)$. Asterisks refer to significant differences between same and different conditions $\left({ }^{*} p<.05 ; * * p<.01\right)$.

models. On some trials, it may have been difficult to tell whether the target was an "L" or a "7," and on others, it may even have been difficult to determine which character was the target, let alone whether it was an "L" or a "7."

\section{DISCUSSION}

The results of the probabilistic modeling, as well as the raw data, demonstrate that a high number of ICs were made in this experiment, replicating the results of previous studies obtaining large IC rates with peripheral presentation. When detecting the letter "L," participants were more likely to incorrectly report the color of the "O" when the flanker characters were also letters, biasing the " $\mathrm{O}$ " to be categorized as a letter. Conversely, when detecting the number " 7 ," participants were more likely to incorrectly report the color of the "O" when the flanker characters were numbers, biasing the " $\mathrm{O}$ " to be categorized as a number. These findings support the hypothesis that semantic category acted to group the characters together, increasing the likelihood of the target and distractor features' being incorrectly conjoined.

This finding indicates that grouping and perhaps object formation are not limited to pure physical characteristics of the stimulus, but also include how the meaning of the stimulus is interpreted. In this case, the " $L$ " and the "7" share the same perceptual features and, in turn, neither shares any perceptual features with the distractor "O." Nevertheless, their meaning alone biased the way in which the visual system bound their features together.

An alternative explanation of the data may be that binding is influenced by prior visual experience. For example, it may be that " 8708 " is a more familiar stimulus, and it is therefore grouped for that reason, rather than for its ho- mogeneity at the abstract categorical level. Given the limited number of stimuli used in the present experiment, such an effect would probably diminish over time throughout the experiment as the stimuli become more familiar. Analyses revealed no change in the categorical effect across blocks $(F<1)$, providing no support for an independent effect of familiarity in this experiment.

In previous experiments, researchers have attempted to find evidence for preattentive processing of alphanumeric category. In a classic visual search study by Jonides and Gleitman (1972), flat reaction time slopes over set size resulted when subjects searched for an "O" among letters when the target was called "zero," whereas increasing slopes resulted when the target was called "oh." This might suggest preattentive processing of a category. In the first case, its "numberness" pops out, whereas in the second, its "letterness" is not unique (Jonides \& Gleitman, 1972). However, subsequent investigation showed that these effects were due to physical similarity; numbers tend to have certain features that are different from letters. When these physical properties were controlled, effects diminished, casting doubt on the notion that early processing of categorical information could guide attention as visual features do (Krueger, 1984). Nevertheless, in a recent visual search study, researchers controlled for perceptual similarity while manipulating categorical similarity by simply varying the names of targets and distractors (Smilek, Dixon, \& Merikle, 2003). Specifically, they established that newly learned semantic associations for random line segments had a significant effect on search rates. Given these findings, together with those of the present study, it appears that processing of meaning can influence binding and the spatial scan of attention. 
A criticism of the present study is that letters and numbers may reflect a special case of semantic processing that is automatic and highly learned. Although more research is needed, the visual search study previously described (Smilek et al., 2003) suggests that even newly learned categorical associations might have a similar influence on grouping and binding. Further studies of ICs will be necessary to determine whether newly learned semantic categories also influence the binding process itself.

\section{REFERENCES}

Arguin, M., Cavanagh, P., \& Joanette, Y. (1994). Visual feature integration with an attentional deficit. Brain \& Cognition, 24, 44-56.

Ashby, F., Prinzmetal, W., Ivry, R., \& Maddox, T. (1996). A formal theory of illusory conjunctions. Psychological Review, 103, 165-192.

Bernstein, L., \& Robertson, L. (1998). Independence between illusory conjunctions of color and motion with shape following bilateral parietal lesions. Psychological Science, 9, 167-175.

CoHEN, A., \& IvrY, R. (1989). Illusory conjunctions inside and outside the focus of attention. Journal of Experimental Psychology: Human Perception \& Performance, 15, 650-653.

CoHEN, A., \& RAFAL, R. (1991). Attention and feature integration: Illusory conjunctions in a patient with a parietal lobe lesion. Psychological Science, 2, 106-110.

Donk, M. (1999). Illusory conjunctions are an illusion: The effects of target-nontarget similarity on conjunction and feature errors. Journal of Experimental Psychology: Human Perception \& Performance, $\mathbf{2 5}$, $1207-1253$.

Friedman-Hill, S., Robertson, L., \& Treisman, A. (1995). Parietal contributions to visual feature binding: Evidence from a patient with bilateral lesions. Science, 269, 853-855.

IVRY, R. B., \& PRINZMETAL, W. (1991). Effect of feature similarity on illusory conjunctions. Perception \& Psychophysics, 49, 105-116.

Jonides, J., \& Gleitman, H. (1972). A conceptual category effect in visual search: $\mathrm{O}$ as a letter or digit. Perception \& Psychophysics, 12, 457-460.

Krueger, L. E. (1984). The category effect in visual search depends on physical rather than conceptual differences. Perception \& Psychophysics, 35, 558-564.

PrinZMetaL, W. (1981). Principles of feature integration in visual perception. Perception \& Psychophysics, 30, 330-340.

Prinzmetal, W., DiedrichSEN, J., \& IVRY, R. (2001). Illusory conjunctions are alive and well: A reply to Donk (1999). Journal of Experimental Psychology: Human Perception \& Performance, 27, 538-541.

Prinzmetal, W., Henderson, D., \& Ivry, R. (1995). Loosening the constraints on illusory conjunctions: Assessing the roles of exposure duration and attention. Journal of Experimental Psychology: Human Perception \& Performance, 21, 1362-1375.

Prinzmetal, W., Hoffman, H., \& Vest, K. (1991). Automatic processes in word perception: An analysis from illusory conjunctions. Journal of Experimental Psychology: Human Perception \& Performance, 17, 902-923.

Prinzmetal, W., IvrY, R., Beck, D., \& Shimizu, N. (2002). A measurement theory of illusory conjunctions. Journal of Experimental Psychology: Human Perception \& Performance, 28, 251-269.

Prinzmetal, W., \& KeYSAR, B. (1989). A functional theory of illusory conjunctions and neon colors. Journal of Experimental Psychology: General, 118, 165-190.

PrinZmetal, W., \& Millis-Wright, M. (1984). Cognitive and linguistic factors affect visual feature integration. Cognitive Psychology, 16, 305-340.

Robertson, L., Treisman, A., Friedman-Hill, S., \& Grabowecky, M. (1997). The interaction of spatial and object pathways: Evidence from Balint"s syndrome. Journal of Cognitive Neuroscience, 9, 295-317.

Smilek, D., Dixon, M., \& MerikLe, P. (2003, November). Meaning influences search efficiency. Poster presented at the annual meeting of the Psychonomic Society, Vancouver, BC.

Treisman, A. (1988). Features and objects: The fourteenth Bartlett Memorial Lecture. Quarterly Journal of Experimental Psychology, 40A, 201-237.

Treisman, A., \& Schmidt, H. (1982). Illusory conjunctions in the perception of objects. Cognitive Psychology, 14, 107-141.

Ward, R., Danziger, S., Owen, V., \& RAFAL, R. (2002). Deficits in spatial coding and feature binding following damage to spatiotopic maps in the human pulvinar. Nature Neuroscience, 5, 99-100.

Wolfe, J., \& Bennetr, S. (1997). Preattentive object files: Shapeless bundles of features. Vision Research, 37, 25-43.

\section{NOTE}

1. The model used in this study is identical to the basic model presented in Prinzmetal et al. (2002; Figure 1), except that the guessing parameter $(g)$ was set to zero. This parameter was used to estimate the probability of guessing the target color to be the same as the distractor color, when only the distractor color was perceived. When all displays contained two different colors, as in the present experiment, $g$ was estimated as zero. Guessing was still accounted for in the model such that each color and target was equally likely to be guessed when the corresponding feature was not perceived.

(Manuscript received April 23, 2003; revision accepted for publication August 11, 2003.) 